\title{
BERGMAN KERNELS OF ELEMENTARY REINHARDT DOMAINS
}

\author{
DEBRAJ CHAKRABARTI, AUSTIN KONKEL, MEERA MAINKAR, AND EVAN MILLER
}

\begin{abstract}
We study the Bergman kernel of certain domains in $\mathbb{C}^{n}$, called elementary Reinhardt domains, generalizing the classical Hartogs triangle. For some elementary Reinhardt domains, we explicitly compute the kernel, which is a rational function of the coordinates. For some other such domains, we show that the kernel is not a rational function. For a general elementary Reinhardt domain, we obtain a representation of the kernel as an infinite series.
\end{abstract}

\section{INTRODUCTION}

1.1. Elementary Reinhardt domains. Let $\mathbb{D}^{n}=\left\{z \in \mathbb{C}^{n}|| z_{j} \mid<1\right.$ for $\left.1 \leq j \leq n\right\}$ denote the unit polydisc in $\mathbb{C}^{n}, n \geq 2$, and let $k=\left(k_{1}, \ldots, k_{n}\right) \in \mathbb{Z}^{n}$ be a multi-index. The goal of this paper is the study of the Bergman kernel of the domain

$$
\mathscr{H}(k)=\left\{z \in \mathbb{D}^{n} \mid z^{k} \text { is defined, and }\left|z^{k}\right|<1\right\},
$$

where we use the standard multi-index convention $z^{k}=z_{1}^{k_{1}} z_{2}^{k_{2}} \ldots z_{n}^{k_{n}}$, and the only way this can fail to be defined is if its evaluation involves division by zero. We will call the domain $\mathscr{H}(k)$ the elementary Reinhardt domain associated to the multi-index $k$ (cf. [JP08, pp.33 ff.], where this terminology is used, with a slightly different definition). A famous example of such a domain is the Hartogs triangle

$$
\mathscr{H}(1,-1)=\left\{\left|z_{1}\right|<\left|z_{2}\right|<1\right\} \subset \mathbb{C}^{2},
$$

a well-known source of counterexamples in several complex variables (see, e.g., [Sha15]).

It is easy to see that $\mathscr{H}(k)$ is logarithmically convex, and therefore pseudoconvex (see [Ran86]). If the multi-index $k$ contains both positive and negative entries, then $\mathscr{H}(k)$ is a Reinhardt domain with the origin as a boundary point, so it follows (see [Cha18]) that each holomorphic function smooth up to the boundary on $\mathscr{H}(k)$ extends to a larger, fixed domain, a property which is classical in the special case of the Hartogs triangle (see [Sib75, Beh33]). Therefore, $\mathscr{H}(k)$ does not have a basis of Stein neighborhoods, and is not a so-called $\mathcal{H}^{\infty}$-domain of holomorphy. This makes domains such as $\mathscr{H}(k)$ particularly interesting from the point of view of function theory on non-smooth domains, since each smoothly bounded pseudoconvex domain is in fact an $\mathcal{H}^{\infty}$-domain of holomorphy ([Cat80, HS80]).

Recently, the unusual $L^{p}$-mapping properties of the Bergman projection on the generalized Hartogs triangle $\mathscr{H}(m,-n) \subset \mathbb{C}^{2}$ (where $m, n$ are coprime positive integers) have received the attention of several authors (see [CZ16, Edh16, EM16, EM17, CEM19]). In many of these investigations, the explicit form of the Bergman kernel of $\mathscr{H}(m,-n) \subset \mathbb{C}^{2}$ plays a crucial role. The elementary Reinhardt domains are a natural class generalizing the Hartogs triangle. Motivated by this, in this

2010 Mathematics Subject Classification. 32A25, 32A07.

Debraj Chakrabarti, Austin Konkel and Evan Miller were partially supported by NSF grant DMS-1600371. Austin Konkel was also supported by a Student Research and Creative Endeavors grant from Central Michigan University. 
paper, we make a preliminary study of the Bergman kernels of the domains $\mathscr{H}(k)$. In particular, we investigate whether such a Bergman kernel is a rational function of the coordinates, as it indeed is if $n=2$ (see [Edh16, EM17]). Other recent attempts at higher dimensional generalizations may be found in Par18, Che17, Huo18, CKY19. For planar domains, the rationality (or algebraicity) of the Bergman kernel has important function-theoretic repercussions (see [Bel05]). It would be interesting to see whether something similar is true for the elementary Reinhardt domains.

From now on we will assume that the multi-index $k=\left(k_{1}, \ldots, k_{n}\right)$ defining the domain (1.1) has the following properties:

(1) At least one of the components of the multi-index is positive, at least one of the components is negative, and no component is zero.

We will call the number of positive components of $k$, the signature $s$ of the elementary Reinhardt domain $\mathscr{H}(k)$.

(2) If $\mathscr{H}(k)$ has signature $s$, after renaming the coordinates, we will assume without loss of generality that $k_{j}>0$ for $1 \leq j \leq s$ and $k_{j}<0$ if $s+1 \leq j \leq n$.

(3) We will also assume without loss of generality that the numbers $k_{1}, \ldots, k_{n}$ are relatively prime.

1.2. Explicit Formula. For elementary Reinhardt domains of signature 1, we now give an explicit formula for the Bergman kernel as a rational function of the coordinates.

To state the result, introduce the following notation. For integers $\lambda$ and $\mu$, let

$$
\mathrm{D}_{\lambda}(\mu)= \begin{cases}0 & \mu \leq-1 \text { or } \mu \geq 2 \lambda-1 \\ \mu+1 & 0 \leq \mu \leq \lambda-1 \\ 2 \lambda-1-\mu & \lambda \leq \mu \leq 2 \lambda-2\end{cases}
$$

It will be seen in Section 4.2 below, that the seemingly complicated expression $D_{\lambda}(\mu)$ arises as the number of solutions in pairs of integers $(x, y)$ of the equation $x+y=\mu$ subject to the constraints $0 \leq x, y \leq \lambda-1$ (see (4.23), (4.24), (4.25) $)$.

Theorem 1.1. Let $n \geq 2$, let $k_{1}, \ldots, k_{n}$ be relatively prime positive integers, and let

$$
k=\left(k_{1},-k_{2}, \ldots,-k_{n}\right) \in \mathbb{Z}^{n}
$$

be a multi-index. The Bergman kernel of the elementary Reinhardt domain $\mathscr{H}(k)$ is given by:

$$
\mathbb{B}_{\mathscr{H}(k)}(z, w)=\frac{1}{\pi^{n} L} \cdot \frac{\sum_{\beta \in \mathfrak{G}} C(\beta) t^{\beta}}{\left(\prod_{b=2}^{n} t_{b}^{k_{b}}-t_{1}^{k_{1}}\right)^{2} \cdot \prod_{b=2}^{n}\left(1-t_{b}\right)^{2}}
$$

where $t=\left(t_{1}, \ldots, t_{n}\right) \in \mathbb{C}^{n}$ with $t_{a}=z_{a} \overline{w_{a}}$ for $1 \leq a \leq n$, and

$$
C(\beta)=\mathrm{D}_{K}\left(2 K-\ell_{1}\left(\beta_{1}+1\right)-1\right) \cdot \prod_{b=2}^{n} \mathrm{D}_{\ell_{b}}\left(\ell_{b}\left(\beta_{b}+1\right)+\ell_{1}\left(\beta_{1}+1\right)-2 K-1\right),
$$

where the function $\mathrm{D}_{*}(\cdot)$ is defined in (1.2) above, with

$$
K=\operatorname{lcm}\left(k_{1}, \ldots, k_{n}\right), \quad \ell_{a}=\frac{K}{k_{a}} \quad \text { for } 1 \leq a \leq n, \text { and } \quad L=\prod_{a=1}^{n} \ell_{a},
$$


and where the finite collection of multi-indices $\mathfrak{G} \subset \mathbb{Z}^{n}$ is defined by

$$
\mathfrak{G}=\left\{\left(\beta_{1}, \ldots, \beta_{n}\right) \in \mathbb{Z}^{n} \mid 0 \leq \beta_{1} \leq 2 k_{1}-2 \text {, and } 0 \leq \beta_{b} \leq 2 k_{b} \text { for each } 2 \leq b \leq n\right\} .
$$

While the expression (1.3) is somewhat complicated, it generalizes and extends several known results in the literature. In the special case of the classical Hartogs triangle $\mathscr{H}(1,-1)$, an explicit expression for the Bergman kernel is already found in [Bre55]. Recently, in [Edh16], Edholm computed using Bell's formula (see (4.2) below) the Bergman kernels of $\mathscr{H}(1,-k)$ and $\mathscr{H}(k,-1)$, where $k \geq 2$ is an integer ("Fat and thin generalized Hartogs triangles"), a computational tour de force which inspired Theorem 1.1. In EM17], Edholm and McNeal studied $\mathscr{H}(m,-n) \subset \mathbb{C}^{2}$, where $m, n$ are coprime positive integers, and expressed its Bergman kernel as the sum of $m$ "sub-Bergman kernels." The sub-Bergman kernels are obtained by summing subseries of the power series (2.2) representing the Bergman kernel of a Reinhardt domain. These subseries consist of terms with monomials whose exponents are represented by straight lines of different slopes in the lattice point diagram of monomials, resulting in a decomposition of the kernel into convenient pieces, which permits the explicit summation of each of the sub-kernels in closed form as a rational function, and determination of the $L^{p}$-regularity of each piece. However, our formula (1.3) shows that splitting the kernel into the sub-kernels is unnecessary, and the main $L^{p}$ estimate of [EM17] could proceed directly from (1.3). Starting from (1.3), we recapture below in Section 4.3 the special cases considered in [Edh16]. Theorem 1.1 also opens the way to generalize the interesting recent results related to $L^{p}$-regularity of the Bergman projection, duality of Bergman spaces etc. (cf. [EM17, CZ16, CEM19]) to higher dimensions.

1.3. Signatures greater than 1. In signatures $s \geq 2$ (so that the ambient dimension $n \geq 3$ ) the situation is much less clear, and is worth further study. Here we collect a few observations which seems to indicate that there are some fundamental differences between the cases $s=1$ and $s \geq 2$. In particular, it seems plausible that the Bergman kernels of elementary Reinhardt domains of signature $s \geq 2$ can not be represented using a simple rational function such as (1.3).

Let $n \geq 2$, and let $1 \leq s \leq n-1$. We denote by $\Omega_{n, s}$ the elementary Reinhardt domain of signature $s$ in $\mathbb{C}^{n}$, where each component of the defining multi-index is \pm 1 , i.e.

$$
\Omega_{n, s}=\mathscr{H}(\underbrace{1, \ldots, 1}_{s}, \underbrace{-1, \ldots,-1}_{n-s}),
$$

so that $\Omega_{n, s}=\left\{z \in \mathbb{D}^{n}|| z_{1} \ldots z_{s}|<| z_{s+1} z_{s+2} \ldots z_{n} \mid\right\}$. We will call $\Omega_{n, s}$ the model elementary domain of signature $s$. It is shown in Proposition 2.1 below that the model elementary domains are branched covers of all elementary Reinhardt domains.

In Theorem 3.1 below, we give an account of the coefficients of the power series expansion of $\mathbb{B}_{\Omega_{n, s}}$ by computing the $L^{2}$-norms of monomials $e_{\alpha}(z)=z_{1}^{\alpha_{1}} \ldots z_{n}^{\alpha_{n}}$. This shows that the coefficient of

$$
\left(z_{1} \overline{w_{1}}\right)^{\alpha_{1}} \ldots\left(z_{n} \overline{w_{n}}\right)^{\alpha_{n}}
$$

in the power series expansion of $\mathbb{B}_{\Omega_{n, s}}$ is a polynomial in $\alpha_{1}, \ldots, \alpha_{n}$ only if $s=1$. For $2 \leq s \leq n-1$, the coefficient is a rational function of $\alpha_{1}, \ldots, \alpha_{n}$. From this we are able to deduce the following:

Theorem 1.2. If $n \geq 3$, then $\mathbb{B}_{\Omega_{n, n-1}}$ is not a rational function.

It seems highly plausible that in fact $\mathbb{B}_{\Omega_{n, s}}$ is a transcendental function of the coordinates unless $s=1$, though at present we are not in possession of a complete proof. If this conjecture is 
correct, using the proper map from a model domain to an arbitrary elementary Reinhardt domain, it will follow that the Bergman kernel of an elementary Reinhardt domain of signature $s \geq 2$ is transcendental.

Some further properties of the series representation of the Bergman kernel are explored in section 3.2 below.

1.4. Acknowledgements. We gratefully acknowledge the helpful comments of Doron Zielberger, Jeff McNeal and Luke Edholm. We also thank the anonymous referee for many excellent suggestions which led to significant improvements.

\section{Preliminaries}

2.1. Bergman theory. We briefly recall some basic facts about Bergman spaces and kernels and clarify our notation. An extensive modern exposition of this topic from the complex analysis point of view is [Kra13, and from the operator theory point of view is [DS04].

Let $\Omega \subset \mathbb{C}^{n}$ be a domain, i.e. a connected open set. Then $A^{2}(\Omega)$, the $\left(L^{2}\right)$-Bergman space of $\Omega$, is the Hilbert space of holomorphic functions which are square integrable with respect to the Lebesgue measure $d V$. This is a so-called reproducing kernel Hilbert space, and its reproducing kernel is the Bergman kernel, a function $\mathbb{B}_{\Omega}: \Omega \times \Omega \rightarrow \mathbb{C}$, holomorphic in the first and anti-holomorphic in the second input such that for each $f \in A^{2}(\Omega)$ we have for each $z \in \Omega$ the reproducing property:

$$
f(z)=\int_{\Omega} f(w) \mathbb{B}_{\Omega}(z, w) d V(w) .
$$

A domain $\Omega \subset \mathbb{C}^{n}$ is Reinhardt if whenever $z \in \Omega$, and $\lambda \in \mathbb{T}^{n}$, where $\mathbb{T}^{n}=\left\{\lambda \in \mathbb{C}^{n}|| \lambda_{j} \mid=\right.$ 1 for each $1 \leq j \leq n\}$ is the unit torus, we have $\left(\lambda_{1} z_{1}, \ldots, \lambda_{n} z_{n}\right) \in \Omega$. For a Reinhardt domain, there is a canonical series representation of the Bergman kernel. For each multi-index $\alpha \in \mathbb{Z}^{n}$, let $e_{\alpha}$ denote the monomial

$$
e_{\alpha}(z)=z^{\alpha}=z_{1}^{\alpha_{1}} \ldots z_{n}^{\alpha_{n}} .
$$

Then the Bergman kernel of $\Omega$ has the series representation converging uniformly on compact subsets of $\Omega \times \Omega$ :

$$
\mathbb{B}_{\Omega}(z, w)=\sum_{\alpha \in \mathbb{Z}^{n}} \frac{1}{\left\|e_{\alpha}\right\|^{2}} z^{\alpha} \overline{w^{\alpha}}
$$

where

$$
\left\|e_{\alpha}\right\|^{2}=\int_{\Omega}\left|e_{\alpha}(z)\right|^{2} d V(z)
$$

and if for an $\alpha \in \mathbb{Z}^{n}$ the integral (2.3) diverges, the coefficient $\frac{1}{\left\|e_{\alpha}\right\|^{2}}$ in (2.2) is taken to be zero. An immediate consequence of this series representation is the following simple observation: if $\widetilde{\Omega} \subset \mathbb{C}^{n}$ is the domain $\widetilde{\Omega}=\left\{\left(z_{1} \overline{w_{1}}, \ldots, z_{n} \overline{w_{n}}\right) \mid z, w \in \Omega\right\}$, then there is a holomorphic function $\widetilde{B}$ on $\widetilde{\Omega}$ such that $\mathbb{B}_{\Omega}(z, w)=\widetilde{B}\left(z_{1} \overline{w_{1}}, \ldots, z_{n} \overline{w_{n}}\right)$, where for $t \in \widetilde{\Omega}$,

$$
\widetilde{B}(t)=\sum_{\alpha \in \mathbb{Z}^{n}} \frac{1}{\left\|e_{\alpha}\right\|^{2}} t^{\alpha} .
$$

Therefore, the Bergman kernel of a Reinhardt domain $\Omega$ can be thought of as a holomorphic function on a different domain $\widetilde{\Omega}$, and this simplifies its study. 
2.2. Model domains as branched covers. A map $\phi: \mathbb{C}^{n} \rightarrow \mathbb{C}^{n}$ will be said to be of the diagonal type if there are positive integers $\ell_{1}, \ldots, \ell_{n}$ such that

$$
\phi\left(z_{1}, \ldots, z_{n}\right)=\left(z_{1}^{\ell_{1}}, \ldots, z_{n}^{\ell_{n}}\right) .
$$

Proposition 2.1. Let $n \geq 2$ and let $H$ be an elementary Reinhardt domain in $\mathbb{C}^{n}$ of signature $1 \leq s \leq n-1$. Then there is a proper holomorphic map of diagonal type from the model elementary domain $\Omega_{n, s}$ of (1.6) to $H$.

Proof. Let $k=\left(k_{1}, \ldots, k_{s},-k_{s+1}, \ldots,-k_{n}\right)$ be the multi-index such that $H=\mathscr{H}(k)$. Let us set $K=\operatorname{lcm}\left(k_{1}, \ldots, k_{n}\right)$, and let $\ell_{j}=\frac{K}{k_{j}}$. Define the map $\phi$ by (2.5). Then $\phi$ defines a proper holomorphic map from $\mathbb{C}^{n}$ to itself. To show that $\phi$ restricts to a proper map from $\Omega_{n, s}$ to $H$, it suffices to show that $\phi^{-1}(H)=\Omega_{n, s}$. Indeed, if $z \in \mathbb{C}^{n}$ is such that $\phi(z) \in H$, then we have $\left|\phi(z)^{k}\right|<1$. But since

$$
\phi(z)^{k}=\left(z_{1}^{\ell_{1}}\right)^{k_{1}} \ldots\left(z_{s}^{\ell_{s}}\right)^{k_{s}}\left(z_{s+1}^{\ell_{s+1}}\right)^{-k_{s+1}} \ldots\left(z_{n}^{\ell_{n}}\right)^{-k_{n}}=\left(z_{1} \ldots z_{s}\right)^{K}\left(z_{s+1} \ldots z_{n}\right)^{-K},
$$

it follows that $z \in \Omega_{n, s}$ and the result follows.

Definition 2.1. Let $H$ be an elementary Reinhardt domain in $\mathbb{C}^{n}$ of signature $s$. The map $\phi: \Omega_{n, s} \rightarrow H$ given by (2.5) will be referred to as the standard proper map associated with $H$.

Note that there may also be proper holomorphic maps from $\Omega_{n, s}$ to $H$ different from the standard map. And for certain elementary Reinhardt domains, biholomorphic maps can even be found. For example, the map from $\Omega_{2,1}=\left\{\left|z_{1}\right|<\left|z_{2}\right|<1\right\} \subset \mathbb{C}^{2}$ to $\mathscr{H}(m,-n)=\left\{\left|z_{1}\right|^{\frac{m}{n}}<\left|z_{2}\right|<1\right\} \subset \mathbb{C}^{2}$ given by $\left(z_{1}, z_{2}\right) \rightarrow\left(z_{1} z_{2}^{n-1}, z_{2}^{m}\right)$ is a proper holomorphic map different from the standard map, and a biholomorphism if and only if $m=1$.

\section{NORMS OF MONOMIALS}

In the following theorem, we describe the coefficients of the series expansion (2.2) of the Bergman kernel of an elementary Reinhardt domain.

Theorem 3.1. Let $n \geq 2$, let $1 \leq s \leq n-1$, and let $\alpha \in \mathbb{Z}^{n}$. Let $\beta \in \mathbb{Z}^{n}$ be the multi-index $\left(\beta_{1}, \ldots, \beta_{n}\right)$ such that

$$
\beta_{j}=\alpha_{j}+1
$$

Then, on the model domain $\Omega_{n, s}$, we have

(1) $\left\|e_{\alpha}\right\|_{\Omega_{n, s}}^{2}<\infty$ if and only if

$$
\beta_{j}>0 \text { and } \beta_{j}+\beta_{\ell}>0 \text { for } 1 \leq j \leq s \text {, and } s+1 \leq \ell \leq n \text {. }
$$

(2) if $\alpha$ is such that $\left\|e_{\alpha}\right\|_{\Omega_{n, s}}^{2}<\infty$ we have that

$$
\left\|e_{\alpha}\right\|_{\Omega_{n, s}}^{2}=\pi^{n} \cdot \frac{R_{n, s}(\beta)}{S_{n, s}(\beta)},
$$

where $R, S$ are homogeneous polynomials in $n$ variables with integer coefficients, with

$$
S_{n, s}(\beta)=\prod_{j=1}^{s} \beta_{j} \prod_{\substack{1 \leq j \leq s \\ s+1 \leq \ell \leq n}}\left(\beta_{j}+\beta_{\ell}\right)
$$


and $R_{n, s}$ is a homogeneous polynomial of total degree $(n-s)(s-1)$ such that $R_{n, s}$ and $S_{n, s}$ have no common factors. We further have $R_{n, 1}=1$.

Recall that the total degree of a monomial is the sum of exponents of each of the variables.

Proof. Fix an $s \geq 1$, and we prove this by induction on $n$. We will start with the base case of $n=s$, which is not part of the statement of the theorem as stated, but for which the result also holds. Denote by $\mathbb{D}^{s}$ the unit polydisc $\left\{\left|z_{j}\right|<1, j=1, \ldots, s\right\}$ in $\mathbb{C}^{s}$. Notice that

$$
\Omega_{s, s}=\left\{z \in \mathbb{D}^{s}|| z_{1} z_{2} \ldots z_{n} \mid<1\right\}=\mathbb{D}^{s} .
$$

In this case we have for $\alpha \in \mathbb{Z}^{s}$ by direct computation that $\left\|e_{\alpha}\right\|^{2}<\infty$ if and only if

$$
\beta_{j}=\alpha_{j}+1>0, \quad j=1, \ldots, s,
$$

and for such $\alpha$

$$
\left\|e_{\alpha}\right\|_{\mathbb{D}^{s}}^{2}=\pi^{s} \frac{1}{\left(\alpha_{1}+1\right) \ldots\left(\alpha_{s}+1\right)}=\pi^{s} \frac{1}{\beta_{1} \ldots \beta_{s}} .
$$

Therefore (3.1) is satisfied, and if we take $R_{s, s}=1$ and $S_{s, s}=\beta_{1} \ldots \beta_{s}$ then (3.3) is satisfied, and $R_{s, s}$ does have degree $(n-s)(s-1)=(s-s)(s-1)=0$, as needed.

We now proceed by induction. Assume the result is true for some $n \geq s$. For simplicity of notation, let $\mathbf{1}=(1,1, \ldots, 1)$, then we set

$$
\mathcal{D}_{n, s}(\beta)=\frac{1}{\pi^{n}}\left\|e_{\beta-1}\right\|_{\Omega_{n, s}}^{2},
$$

and for $\beta_{n+1} \in \mathbb{Z}$ denote by $\left(\beta, \beta_{n+1}\right) \in \mathbb{Z}^{n+1}$ the multi-index

$$
\left(\beta, \beta_{n+1}\right)=\left(\beta_{1}, \ldots \beta_{n}, \beta_{n+1}\right) .
$$

To abbreviate the formulas that follow, let $\beta^{*} \in \mathbb{Z}^{n}$ be the multi-index given by

$$
\beta_{j}^{*}= \begin{cases}\beta_{j}+\beta_{n+1} & \text { if } 1 \leq j \leq s \\ \beta_{j}-\beta_{n+1} & \text { if } s+1 \leq j \leq n .\end{cases}
$$

Notice that $\beta^{*}$ actually depends on $\beta \in \mathbb{Z}^{n}$ and $\beta_{n+1} \in \mathbb{Z}$, though this has been suppressed from the notation. We claim that $\mathcal{D}_{n+1, k}\left(\beta, \beta_{n+1}\right)$ can be represented as follows

$$
\mathcal{D}_{n+1, s}\left(\beta, \beta_{n+1}\right)=\frac{1}{\beta_{n+1}}\left(\mathcal{D}_{n, s}(\beta)-\mathcal{D}_{n, s}\left(\beta^{*}\right)\right) .
$$

We postpone the proof of the claim to proceed with the induction. Note that $\left\|e_{\beta-1}\right\|<\infty$ is equivalent to $\mathcal{D}_{n, s}(\beta)<\infty$. Let $\left(\beta, \beta_{n+1}\right) \in \mathbb{Z}^{n}$. From (3.7), it follows that $\mathcal{D}_{n+1, s}\left(\beta, \beta_{n+1}\right)<\infty$ if and only if $\mathcal{D}_{n, s}(\beta)<\infty$ and $\mathcal{D}_{n, s}\left(\beta^{*}\right)<\infty$, since each of $\mathcal{D}_{n, s}(\beta)$ and $\mathcal{D}_{n, s}\left(\beta^{*}\right)$ is strictly positive. From $\mathcal{D}_{n, s}(\beta)<\infty$, using the induction hypothesis, we see that the conditions (3.1) hold. From $\mathcal{D}_{n, s}\left(\beta^{*}\right)<\infty$ we get the conditions

$$
\beta_{j}^{*}>0 \text { and } \beta_{j}^{*}+\beta_{\ell}^{*}>0 \text { for } 1 \leq j \leq s \text {, and } s+1 \leq \ell \leq n,
$$

which, using the definition of $\beta_{j}^{*}$ in (3.6) becomes

$$
\beta_{j}+\beta_{n+1}>0, \quad \text { and } \quad \beta_{j}+\beta_{\ell}>0, \quad 1 \leq j \leq s \text {, and } s+1 \leq \ell \leq n+1 .
$$

Now (3.1) and (3.8) together imply that the conclusion (1) of the theorem we are proving holds for $n+1$, provided it holds for $n$. 
Assuming now that $\mathcal{D}_{n+1, s}\left(\beta, \beta_{n+1}\right)<\infty$, by (3.7), and the induction hypothesis, we have that

$$
\begin{aligned}
\mathcal{D}_{n+1, s}\left(\beta, \beta_{n+1}\right) & =\frac{1}{\beta_{n+1}}\left(\frac{R_{n, s}(\beta)}{S_{n, s}(\beta)}-\frac{R_{n, s}\left(\beta^{*}\right)}{S_{n, s}\left(\beta^{*}\right)}\right) \\
& =\frac{1}{\beta_{n+1}}\left(\frac{R_{n, s}(\beta) S_{n, s}\left(\beta^{*}\right)-R_{n, s}\left(\beta^{*}\right) S_{n, s}(\beta)}{S_{n, s}(\beta) S_{n, s}\left(\beta^{*}\right)}\right) .
\end{aligned}
$$

Using the definition (3.6) of $\beta^{*}$, we have

$$
\begin{aligned}
S_{n, s}\left(\beta^{*}\right) & =\prod_{j=1}^{s} \beta_{j}^{*} \prod_{\substack{1 \leq j \leq s \\
s+1 \leq \ell \leq n}}\left(\beta_{j}^{*}+\beta_{\ell}^{*}\right)=\prod_{j=1}^{s}\left(\beta_{j}+\beta_{n+1}\right) \prod_{\substack{1 \leq j \leq s \\
s+1 \leq \ell \leq n}}\left(\beta_{j}+\beta_{\ell}\right) \\
& =\prod_{\substack{1 \leq j \leq s \\
s+1 \leq \ell \leq n+1}}\left(\beta_{j}+\beta_{\ell}\right) .
\end{aligned}
$$

Therefore using (3.3) and (3.10):

$$
\begin{aligned}
S_{n, s}(\beta) S_{n, s}\left(\beta^{*}\right) & =\prod_{j=1}^{s} \beta_{j} \prod_{\substack{1 \leq j \leq s \\
s+1 \leq \ell \leq n}}\left(\beta_{j}+\beta_{\ell}\right) \prod_{\substack{1 \leq j \leq s \\
s+1 \leq \ell \leq n+1}}\left(\beta_{j}+\beta_{\ell}\right) \\
& =S_{n+1, s}\left(\beta, \beta_{n+1}\right) \cdot \prod_{\substack{1 \leq j \leq s \\
s+1 \leq \ell \leq n}}\left(\beta_{j}+\beta_{\ell}\right),
\end{aligned}
$$

where $S_{n+1, s}\left(\beta, \beta_{n+1}\right)$ is as in (3.3). The expression in the numerator of (3.9) is given, using (3.10) and (3.3) by

$$
\begin{aligned}
& R_{n, s}(\beta) S_{n, s}\left(\beta^{*}\right)-R_{n, s}\left(\beta^{*}\right) S_{n, s}(\beta) \\
& =R_{n, s}(\beta) \cdot \prod_{\substack{1 \leq j \leq s \\
s+1 \leq \ell \leq n+1}}\left(\beta_{j}+\beta_{\ell}\right)-R_{n, s}\left(\beta^{*}\right) \cdot \prod_{j=1}^{s} \beta_{j} \prod_{\substack{1 \leq j \leq s \\
s+1 \leq \ell \leq n}}\left(\beta_{j}+\beta_{\ell}\right) \\
& =\left(R_{n, s}(\beta) \cdot \prod_{j=1}^{s}\left(\beta_{j}+\beta_{n+1}\right)-R_{n, s}\left(\beta^{*}\right) \cdot \prod_{j=1}^{s} \beta_{j}\right) \cdot \prod_{\substack{1 \leq j \leq s \\
s+1 \leq \ell \leq n}}\left(\beta_{j}+\beta_{\ell}\right) .
\end{aligned}
$$

Using (3.11) and (3.12) in (3.9), we see that the numerator and denominator of (3.9) share the common factor $\prod_{\substack{1 \leq j \leq s \\ s+1 \leq \ell \leq n}}\left(\beta_{j}+\beta_{\ell}\right)$. Removing this common factor we see that

$$
\mathcal{D}_{n+1}\left(\beta, \beta_{n+1}\right)=\frac{f\left(\beta_{n+1}\right) /\left(\beta_{n+1}\right)}{S_{n+1, s}\left(\beta, \beta_{n+1}\right)},
$$

where we now think of $\left(\beta_{1}, \ldots, \beta_{n+1}\right)$ as indeterminates, and $f$ as a polynomial in the $\operatorname{ring} \mathbb{Q}\left(\beta_{1}, \ldots, \beta_{n}\right)\left[\beta_{n+1}\right]$ of polynomials in the indeterminate $\beta_{n+1}$ over the field of rational functions $\mathbb{Q}\left(\beta_{1}, \ldots, \beta_{n}\right)$ in $n$ indeterminates, with $f$ given by

$$
f\left(\beta_{n+1}\right)=R_{n, s}(\beta) \cdot \prod_{j=1}^{s}\left(\beta_{j}+\beta_{n+1}\right)-R_{n, s}\left(\beta^{*}\right) \cdot \prod_{j=1}^{s} \beta_{j} .
$$


Now the formulas (3.6) defining $\beta^{*}$ in terms of $\beta_{1}, \ldots, \beta_{n+1}$ show that if $\beta_{n+1}=0$, then $\beta^{*}=\beta$. It now follows that $f(0)=0$, so that

$$
R_{n+1, s}\left(\beta, \beta_{n+1}\right)=f\left(\beta_{n+1}\right) / \beta_{n+1},
$$

is a polynomial in the ring $\mathbb{Q}\left(\beta_{1}, \ldots, \beta_{n}\right)$. But noting further that $f \in \mathbb{Z}\left[\beta_{1}, \ldots, \beta_{n+1}\right]$, and the divisor $\beta_{n+1}$ has leading coefficient 1 , we see that in fact $R_{n+1, s} \in \mathbb{Z}\left[\beta_{1}, \ldots, \beta_{n+1}\right]$ which we wanted to prove. We therefore have the recursive formula:

$$
R_{n+1, s}\left(\beta, \beta_{n+1}\right)=\frac{1}{\beta_{n+1}} \cdot\left(R_{n, s}(\beta) \cdot \prod_{j=1}^{s}\left(\beta_{j}+\beta_{n+1}\right)-R_{n, s}\left(\beta^{*}\right) \cdot \prod_{j=1}^{s} \beta_{j}\right) .
$$

By the induction hypothesis, $R_{n, s}$ is a homogeneous polynomial in the $n$ variables $\beta_{1}, \ldots, \beta_{n}$ of total degree $(n-s)(s-1)$. By the definition (3.6) of $\beta^{*}$, we see that $R_{n, s}\left(\beta^{*}\right)$ is a also homogeneous polynomial of the $n+1$ variables $\beta_{1}, \ldots, \beta_{n+1}$. The quantity in large parentheses in (3.13) is therefore the difference of two homogeneous polynomials of total degree $(n-s)(s-1)+s$. It is therefore either zero, or itself a homogeneous polynomial of degree $(n-s)(s-1)+s$. But it cannot be zero, since then the norm of a monomial is zero, which is absurd. Finally by (3.13), the polynomial $R_{n+1, s}$ is also homogeneous, being the ratio of two homogeneous polynomials, and has total degree

$$
(n-s)(s-1)+s-1=((n+1)-s)(s-1) .
$$

We will now show that $R_{n+1, s}(\beta)$ and $S_{n+1, s}(\beta)$ have no common factors.

By induction hypothesis $S_{n, s}(\beta)$ has no common factors with $R_{n, s}(\beta)$. Since $S_{n, s}(\beta)$ is a product of linear factors $\beta_{j}$ and $\left(\beta_{j}+\beta_{\ell}\right)$ where $1 \leq j \leq s$, and $s+1 \leq \ell \leq n$, none of these factors divide $R_{n, s}(\beta)$.

From the symmetry of $\Omega_{n+1, s}$, the definition (3.2), and the symmetry of $S_{n+1, s}$ we know that $R_{n+1, s}\left(\beta, \beta_{n}+1\right)$ is symmetric in variables $\beta_{1}, \ldots, \beta_{s}$ and variables $\beta_{s+1}, \ldots, \beta_{n+1}$. Starting from these facts, we can verify that none of the linear factors of $S_{n+1, s}$ divides the right hand side of (3.13), by noting that even if these linear factors vanish, the right hand side of (3.13) does not. Hence, $R_{n+1, s}\left(\beta, \beta_{n+1}\right)$ and $S_{n+1, s}\left(\beta, \beta_{n+1}\right)$ have no common factors.

Therefore, the inductive proof of the Theorem is complete, except that we need to establish the claim (3.7) on which the above induction was based. Note that from (3.5), with $\mathbf{1}=(1, \ldots, 1)$, we have

$$
\mathcal{D}_{n, s}(\beta)=\frac{1}{\pi^{n}}\left\|e_{\beta-1}\right\|^{2}=\frac{1}{\pi^{n}} \int_{\Omega_{n, s}}\left|e_{\beta-1}(z)\right|^{2} d V(z) .
$$

Using polar coordinates $z_{j}=r_{j} e^{i \theta_{j}}$ and using the fact that $d V(z)=\prod_{j=1}^{n} r_{j} d r_{j} d \theta_{j}=r^{\mathbf{1}} d V(r) d V(\theta)$, where $r=\left(r_{1}, \ldots, r_{n}\right)$, we have

$$
\mathcal{D}_{n, s}(\beta)=\frac{1}{\pi^{n}} \cdot(2 \pi)^{n} \int_{\left|\Omega_{n, s}\right|} r^{2 \beta-1} d V(r),
$$

where $\left|\Omega_{n, s}\right| \subset \mathbb{R}^{n}$ is the Reinhardt shadow of $\Omega_{n, s}$, i.e., the image of $\Omega_{n, s}$ under the map $z \mapsto\left(\left|z_{1}\right|, \ldots,\left|z_{n}\right|\right)$. We will make the further change of variables $t_{j}=r_{j}^{2}$, which maps $\left|\Omega_{n, s}\right|$ diffeomorphically to itself. The integral now takes the form:

$$
\mathcal{D}_{n, s}(\beta)=\frac{1}{\pi^{n}} \cdot(2 \pi)^{n} \int_{\left|\Omega_{n, s}\right|} t^{\beta-1} d V(t) .
$$


We will transform this integral into an $n$-fold repeated integral. For simplicity of notation we denote repeated integrals with differential in front and integrand after that, so that

$$
\int_{x_{2}=a_{2}}^{b_{2}} g\left(x_{2}\right)\left(\int_{x_{1}=a_{1}}^{b_{1}} f\left(x_{1}, x_{2}\right) d x_{1}\right) d x_{2}=\int_{a_{2}}^{b_{2}} d x_{2} \cdot g\left(x_{2}\right) \int_{a_{1}}^{b_{1}} d x_{1} \cdot f\left(x_{1}, x_{2}\right),
$$

and adopt similar notations for multiple repeated integrals, so that the innermost integral in the conventional notation is the rightmost factor. The region of integration over which $t \in \mathbb{R}^{n}$ ranges is described by the inequalities

$$
0 \leq t_{1} \ldots t_{s}<t_{s+1} \ldots t_{n}<1,0 \leq t_{1}<1, \ldots, 0 \leq t_{n}<1 .
$$

Then, $\mathcal{D}_{n, s}(\beta)$ can be expressed explicitly by the following $n$-fold integral:

$\int_{0}^{1} d t_{1} \cdot t_{1}^{\beta_{1}-1} \int_{0}^{1} d t_{2} \cdot t_{2}^{\beta_{2}-1} \cdots \int_{0}^{1} d t_{s} \cdot t_{s}^{\beta_{s}-1} \int_{t_{1} \ldots t_{s}}^{1} d t_{s+1} \cdot t_{s+1}^{\beta_{s+1}-1} \int_{\frac{t_{1} \ldots t_{s}}{t_{s+1}}}^{1} d t_{s+2} \cdot t_{s+2}^{\beta_{s+2}-1} \cdots \int_{\frac{t_{1} \ldots t_{s}}{t_{s+1} \ldots t_{n-1}}}^{1} d t_{n} \cdot t_{n}^{\beta_{n}-1}$.

Similarly,

$$
\begin{aligned}
\mathcal{D}_{n+1, s}\left(\beta, \beta_{n+1}\right)= & \int_{0}^{1} d t_{1} \cdot t_{1}^{\beta_{1}-1} \ldots \int_{0}^{1} d t_{s} t_{s}^{\beta_{s}-1} \int_{t_{1} \ldots t_{s}}^{1} d t_{s+1} t_{s+1}^{\beta_{s+1}-1} \int_{\frac{t_{1} \ldots t_{s}}{t_{s+1}}}^{1} d t_{s+2} t_{s+2}^{\beta_{s+2}-1} \ldots \\
& \cdots \int_{\frac{t_{1} \ldots t_{s}}{t_{s+1} \ldots t_{n-1}}}^{1} d t_{n} t_{n}^{\beta_{n}-1} \int_{\frac{t_{1} \ldots t_{s}}{t_{s+1} \ldots t_{n}}}^{1} d t_{n+1} t_{n+1}^{\beta_{n+1}-1} \\
& =\int_{0}^{1} d t_{1} t_{1}^{\beta_{1}-1} \ldots \int_{0}^{1} d t_{s} t_{s}^{\beta_{s}-1} \int_{t_{1} \ldots t_{s}}^{1} d t_{s+1} t_{s+1}^{\beta_{s+1}-1} \int_{\frac{t_{1} \cdot t_{s}}{t_{s+1}}}^{1} d t_{s+2} \cdot t_{s+2}^{\beta_{s+2}-1} \ldots \\
& \cdots \int_{\frac{t_{1} \ldots t_{s}}{t_{s+1} \ldots t_{n-1}}}^{1} d t_{n} \cdot \frac{1}{\beta_{n+1}}\left(1-\left(\frac{t_{1} \ldots t_{s}}{t_{s+1} \ldots t_{n}}\right)^{\beta_{n+1}}\right)
\end{aligned}
$$

(where we have evaluated the innermost integral)

$$
\begin{aligned}
& =\frac{1}{\beta_{n+1}}\left(\mathcal{D}_{n, s}(\beta)-\right. \\
& \int_{0}^{1} d t_{1} \cdot t_{1}^{\beta_{1}+\beta_{n+1}-1} \ldots \int_{0}^{1} d t_{s} t_{s}^{\beta_{s}+\beta_{n+1}-1} \int_{t_{1} \ldots t_{s}}^{1} d t_{s+1} t_{s+1}^{\beta_{s+1}-\beta_{n+1}-1} \int_{\frac{t_{1} \ldots t_{s}}{t_{s+1}}}^{1} d t_{s+2} t_{s+2}^{\beta_{s+2}-\beta_{n+1}-1} \\
& \left.\cdots \int_{\frac{t_{1} \ldots t_{s}}{t_{s+1} \ldots t_{n-1}}}^{1} d t_{n} \cdot t_{n}^{\beta_{n}-\beta_{n+1}-1}\right) \\
& =\frac{1}{\beta_{n+1}}\left(\mathcal{D}_{n, s}(\beta)-\mathcal{D}_{n, s}\left(\beta^{*}\right)\right)
\end{aligned}
$$

which completes the proof of (3.7).

3.1. Proof of Theorem 1.2, From (2.2), we may write

$$
\mathbb{B}_{\Omega_{n, n-1}}(z, w)=\sum_{\beta \in \mathcal{T}} \frac{1}{\left\|e_{\beta-1}\right\|^{2}} t^{\beta-1}
$$


where $\mathbf{1}=(1, \ldots, 1)$ and $\mathcal{T}$ is the set of indices corresponding to $s=n-1$ in (3.1), i.e.

$$
\beta_{j}>0, \beta_{j}+\beta_{n}>0, \text { for } 1 \leq j \leq n-1 .
$$

Furthermore, we have from Theorem 3.1 that

$$
\left\|e_{\beta-1}\right\|^{2}=\pi^{n} \frac{R_{n, n-1}(\beta)}{S_{n, n-1}(\beta)},
$$

where by (3.3), we have

$$
S_{n, n-1}(\beta)=\prod_{j=1}^{n-1} \beta_{j} \prod_{j=1}^{n-1}\left(\beta_{j}+\beta_{n}\right)=\prod_{j=1}^{n-1} \beta_{j}\left(\beta_{j}+\beta_{n}\right),
$$

and using the recursive relation (23.13) and the fact that $R_{n, n} \equiv 1$ (see (3.4)), we see that

$$
R_{n, n-1}(\beta)=\frac{1}{\beta_{n}}\left(\prod_{j=1}^{n-1}\left(\beta_{j}+\beta_{n}\right)-\prod_{j=1}^{n-1} \beta_{j}\right) .
$$

Therefore, with $t_{j}=z_{j} \overline{w_{j}}$, we have

$$
\begin{aligned}
\mathbb{B}_{\Omega_{n, n-1}}(z, w) & =\widetilde{B}\left(t_{1}, \ldots, t_{n}\right) \\
& =\frac{1}{\pi^{n}} \sum_{\beta \in \mathcal{T}} \frac{\prod_{j=1}^{n-1} \beta_{j}\left(\beta_{j}+\beta_{n}\right)}{\frac{1}{\beta_{n}}\left(\prod_{j=1}^{n-1}\left(\beta_{j}+\beta_{n}\right)-\prod_{j=1}^{n-1} \beta_{j}\right)} t^{\beta-1} .
\end{aligned}
$$

We now consider the function $\widetilde{b}$ of one variable defined by

$$
\widetilde{b}\left(t_{n}\right)=\widetilde{B}(\underbrace{0, \ldots, 0}_{n-1}, t_{n}) .
$$

This is defined in the punctured disc $\left\{0<\left|t_{n}\right|<1\right\}$, and noting that in (3.14) only the terms with $\beta_{j}=1,1 \leq j \leq n-1$ survive if $t_{1}=\cdots=t_{n-1}=0$, we conclude that

$$
\begin{aligned}
\widetilde{b}\left(t_{n}\right) & =\sum_{\beta_{n}=0}^{\infty} \frac{\beta_{n}\left(1+\beta_{n}\right)^{n-1}}{\left(1+\beta_{n}\right)^{n-1}-1} t_{n}^{\beta_{n}-1} \\
& =\frac{t_{n}^{-1}}{n-1}+\sum_{k=1}^{\infty} k t_{n}^{k-1}+\sum_{k=1}^{\infty} \frac{k}{(k+1)^{n-1}-1} t_{n}^{k-1} \\
& =\frac{t_{n}^{-1}}{n-1}+\frac{1}{\left(1-t_{n}\right)^{2}}+\widehat{b}\left(t_{n}\right)
\end{aligned}
$$

where

$$
\widehat{b}\left(t_{n}\right)=\sum_{k=1}^{\infty} \frac{k}{(k+1)^{n-1}-1} \cdot t_{n}^{k-1}
$$

Since the function $\widetilde{B}$ is holomorphic on the domain $\left\{\left(z_{1} \overline{w_{1}}, \ldots, z_{n} \overline{w_{n}}\right) \mid z, w \in \Omega_{n, n-1}\right\}$ it follows that $\widetilde{b}$ is holomorphic in the punctured disc $\left\{0<\left|t_{n}\right|<1\right\}$, and therefore $\widehat{b}$ is holomorphic in the unit disc $\left\{\left|t_{n}\right|<1\right\}$. 
Now for a contradiction, assume that $\mathbb{B}_{\Omega_{n, n-1}}$ is rational. It follows that $\widehat{b}$ is a rational function of one variable, holomorphic in the unit disc, and its $k$-th Taylor coefficient decays as $k^{-(n-2)}$ as $k \rightarrow \infty$. Recall that by hypothesis $n \geq 3$, so the coefficients go to zero.

Let $\alpha_{1}, \ldots, \alpha_{m}$ be the poles of the rational function $\widehat{b}$, where $\left|\alpha_{j}\right| \geq 1$ since $\widehat{b}$ is holomorphic in the unit disc. It follows by expansion in partial fractions (see [FS09, p. 256ff]) that the $k$-th Taylor coefficient of $\widehat{b}$ is of the form $\sum_{j=1}^{m} \alpha_{j}^{-k} \Pi_{j}(k)$ where $\Pi_{j}$ is a polynomial for each $j$. Since the coefficients go to zero as $k \rightarrow \infty$, we must have $\left|\alpha_{j}\right|>1$, for each $j=1, \ldots, m$. Therefore, the decay of the coefficients is exponential in $k$, which contradicts the $k^{-(n-2)}$ decay. Therefore $\widehat{b}$ cannot be a rational function, and so $\mathbb{B}_{\Omega_{n, n-1}}$ is not a rational function if $n \geq 3$.

3.2. Some remarks on the nature of the Bergman Kernel of $\Omega_{n, s}$. The form of the coefficients of the series in Theorem 3.1 as well as the argument in the proof of Theorem 1.2 suggest that the Bergman kernel of $\Omega_{n, s}$ is not rational except for $s=1$, though we do not have a complete proof of this yet. However, Theorem 3.1 is already sufficient to rule out certain hasty conjectures about the form of $\mathbb{B}_{\Omega_{n, s}}$ that one might make based on (1.3) or similar formulas in [Par18]. For example, for $s \neq 1$, the kernel $\mathbb{B}_{\Omega_{n, s}}$ cannot be written in the form

$$
\frac{1}{\pi^{n}} \frac{P(t)}{\left(\prod_{b=s+1}^{n} t_{b}^{k_{b}}-\prod_{a=1}^{s} t_{a}^{k_{a}}\right)^{2} \cdot \prod_{b=s+1}^{n}\left(1-t_{b}\right)^{2}},
$$

for a polynomial $P$, since the coefficient of $t^{\alpha}$ of the Taylor expansion of this function is a polynomial in $\alpha$. Additionally, we saw above that when $s \neq 1$, the Taylor coefficients of $\mathbb{B}_{\Omega_{n, s}}$ are rational functions of $\alpha$ which are not polynomials. Another interesting algebraic property is given by the following:

Proposition 3.1. Let $n \geq 2$ and $1 \leq s \leq n-1$. Let $\widetilde{B}$ be the function of $t_{j}=z_{j} \overline{w_{j}}$ associated with the Bergman kernel of $\Omega_{n, s}$, as defined in (2.4). Then there is a nonzero linear differential operator $\mathscr{L}$ with polynomial coefficients, such that $\mathscr{L} \widetilde{B}$ is a polynomial.

Proof. The case $s=1$ is trivial, since then by Theorem 1.1, $\widetilde{B}$ is a rational function $P / Q$, where $P, Q$ are polynomials. Therefore we can simply take $\mathscr{L}$ to be the zeroth order multiplication operator determined by $Q$.

Notice that we can write, thanks to Theorem 3.1, the series representation

$$
\widetilde{B}(t)=\frac{1}{\pi^{n}} \cdot \frac{1}{t_{1} \ldots t_{n}} \sum_{\beta \in \mathcal{S}} \frac{S_{n, s}(\beta)}{R_{n, s}(\beta)} t^{\beta},
$$

where $R_{n, s}\left(\beta_{1}, \ldots, \beta_{n}\right)$ and $S_{n, s}\left(\beta_{1}, \ldots, \beta_{n}\right)$ are homogeneous polynomials in the variables $\beta_{1}, \ldots, \beta_{n}$, and $\mathcal{S}$ is the subset of $\mathbb{Z}^{n}$ determined by the conditions (3.1). Let $\mathcal{M}$ denote the multiplication operator induced by the polynomial $t_{1} \ldots t_{n}$, and let

$$
\mathscr{L}_{0}=R_{n, s}\left(t_{1} \frac{\partial}{\partial t_{1}}, \ldots, t_{n} \frac{\partial}{\partial t_{n}}\right) \circ \mathcal{M}
$$

Then we see that

$$
\mathscr{L}_{0} \widetilde{B}(t)=\frac{1}{\pi^{n}} \sum_{\beta \in S} S_{n, s}(\beta) t^{\beta}
$$


Now since the coefficients $S_{n, s}(\beta)$ are polynomials in $\beta$ and the region of summation $\mathcal{S}$ is the intersection of a finite number of closed half-spaces in $\mathbb{Z}^{n}$ (since the open conditions in (3.1) can be replaced by closed conditions), it follows that the right hand side of (3.16) is a rational function (cf. the proof of Theorem 1.1 below). If $Q(t)$ is the denominator of this rational function, and $\mathcal{Q}$ is the multiplication operator induced by $Q$, we can take $\mathscr{L}=\mathcal{Q} \circ \mathscr{L}_{0}$.

\section{Proof of Theorem 1.1}

4.1. Kernel of Model domain. We begin by computing the Bergman kernel of the model elementary Reinhardt domain $\Omega_{n, 1}$ :

Proposition 4.1. The Bergman kernel of $\Omega_{n, 1}$ is given by

$$
\mathbb{B}_{\Omega_{n, 1}}(z, w)=\frac{1}{\pi^{n}} \cdot \frac{\prod_{b=2}^{n} t_{b}}{\left(\prod_{b=2}^{n} t_{b}-t_{1}\right)^{2} \cdot \prod_{b=2}^{n}\left(1-t_{b}\right)^{2}},
$$

where

$$
t_{b}=z_{b} \overline{w_{b}} \quad \text { for } 1 \leq b \leq n .
$$

Proof. From Theorem 3.1, we see that for $\alpha \in \mathbb{Z}^{n}$, we have $\left\|e_{\alpha}\right\|_{\Omega_{n, 1}}^{2}<\infty$ if and only if

$$
\alpha_{1}+1>0, \alpha_{1}+\alpha_{\ell}+2>0, \quad 2 \leq \ell \leq n,
$$

which is equivalent to

$$
\alpha_{1} \geq 0, \alpha_{1}+\alpha_{\ell}+1 \geq 0, \quad 2 \leq \ell \leq n .
$$

Let $\mathcal{S} \subset \mathbb{Z}^{n}$ be the set of multi-indices satisfying the above condition. Also from Theorem 3.1, it follows that for $\alpha \in \mathcal{S}$ we have

$$
\left\|e_{\alpha}\right\|_{\Omega_{n, 1}}^{2}=\pi^{n} \frac{1}{\left(\alpha_{1}+1\right) \prod_{b=2}^{n}\left(\alpha_{1}+\alpha_{b}+2\right)} .
$$

Using (2.2) and the abbreviation $t_{b}=z_{b} \overline{w_{b}}$, we have by a direct summation of the series (2.2):

$$
\begin{aligned}
\mathbb{B}_{\Omega_{n, 1}}(z, w) & =\frac{1}{\pi^{n}} \sum_{\alpha \in \mathcal{S}}\left(\left(\alpha_{1}+1\right) \prod_{b=2}^{n}\left(\alpha_{1}+\alpha_{b}+2\right)\right) t^{\alpha} \\
& =\frac{1}{\pi^{n}} \cdot \sum_{\alpha_{1}=0}^{\infty}\left(\alpha_{1}+1\right) t_{1}^{\alpha_{1}} \prod_{b=2}^{n}\left(\sum_{\alpha_{b}=-\alpha_{1}-1}^{\infty}\left(\alpha_{1}+\alpha_{b}+2\right) t_{b}^{\alpha_{b}}\right) \\
& =\frac{1}{\pi^{n}} \cdot \prod_{b=2}^{n} \frac{1}{t_{b}\left(1-t_{b}\right)^{2}} \sum_{\alpha_{1}=0}^{\infty}\left(\alpha_{1}+1\right) t_{1}^{\alpha_{1}} \prod_{b=2}^{n} t_{b}^{-\alpha_{1}} \\
& \left(\text { using the easily proved identity } \sum_{\alpha_{b}=-\alpha_{1}-1}^{\infty}\left(\alpha_{1}+\alpha_{b}+2\right) t_{b}^{\alpha_{b}}=\frac{t_{b}^{-\alpha_{1}-1}}{\left(1-t_{b}\right)^{2}}\right) \\
& =\frac{1}{\pi^{n}} \cdot \prod_{b=2}^{n} \frac{1}{t_{b}\left(1-t_{b}\right)^{2}} \sum_{\alpha_{1}=0}^{\infty}\left(\alpha_{1}+1\right) \rho^{\alpha_{1}}, \quad \text { with } \rho=\frac{t_{1}}{\prod_{b=2}^{n} t_{b}}
\end{aligned}
$$




$$
\begin{aligned}
& =\frac{1}{\pi^{n}} \cdot \frac{1}{(1-\rho)^{2}} \cdot \prod_{b=2}^{n} \frac{1}{t_{b}\left(1-t_{b}\right)^{2}} \\
& =\frac{1}{\pi^{n}} \cdot \frac{1}{\left(1-\frac{t_{1}}{\prod_{b=2}^{n} t_{b}}\right)^{2}} \cdot \prod_{b=2}^{n} \frac{1}{t_{b}\left(1-t_{b}\right)^{2}} \\
& =\frac{1}{\pi^{n}} \cdot \frac{\prod_{b=2}^{n} t_{b}}{\left(\prod_{b=2}^{n} t_{b}-t_{1}\right)^{2} \cdot \prod_{b=2}^{n}\left(1-t_{b}\right)^{2}}
\end{aligned}
$$

where we have used the identity $\sum_{\alpha_{1}=0}^{\infty}\left(\alpha_{1}+1\right) \rho^{\alpha_{1}}=\frac{1}{(1-\rho)^{2}}$ which holds since $|\rho|<1$.

4.2. Explicit Kernel. The following simple arithmetical fact will be used:

Lemma 4.2. Let $k_{1}, \ldots, k_{n}$ be positive integers such that $\operatorname{gcd}\left(k_{1}, \ldots, k_{n}\right)=1$, i.e. $k_{1}, \ldots, k_{n}$ are relatively prime. Let $K=\operatorname{lcm}\left(k_{1}, \ldots, k_{n}\right)$ and $\ell_{j}=\frac{K}{k_{j}}$ with $1 \leq j \leq n$. Then

$$
\operatorname{lcm}\left(\ell_{1}, \ldots, \ell_{n}\right)=K
$$

Proof. Let $k_{j}=\prod_{p \in \text { Primes }} p^{v_{j}(p)}$ be the prime factoring of $k_{j}$. Then $K=\prod_{p \in \text { Primes }} p^{N(p)}$ where

$$
N(p)=\max _{1 \leq j \leq n}\left(v_{j}(p)\right)
$$

Now

$$
\ell_{j}=\frac{K}{k_{j}}=\prod_{p \in \text { Primes }} p^{N(p)-v_{j}(p)}
$$

So,

$$
\operatorname{lcm}\left(\ell_{1}, \ldots, \ell_{n}\right)=\prod_{p \in \text { Primes }} p^{\max _{j}\left(N(p)-v_{j}(p)\right)}=\prod_{p \in \text { Primes }} p^{N(p)-\min _{j}\left(v_{j}(p)\right)}=\prod_{p \in \text { Primes }} p^{N(p)}=K,
$$

where we have used the fact that $\operatorname{since} \operatorname{gcd}\left(k_{1}, \ldots, k_{n}\right)=1$, it follows that $\min _{1 \leq j \leq n}\left(v_{j}(p)\right)=0$.

Proof of Theorem 1.1. Let $\phi: \Omega_{n, 1} \rightarrow \mathscr{H}(k)$ be the standard proper holomorphic map which was constructed in Proposition 2.1. Notice that this map is given by the formula

$$
\phi\left(z_{1}, \ldots, z_{n}\right)=\left(z_{1}^{\ell_{1}}, \ldots, z_{n}^{\ell_{n}}\right),
$$

where $\ell_{j}$ has exactly the same meaning as in the statement of our result. Now by the famous Bell transformation formula ([Bel82]):

$$
u(z) \cdot \mathbb{B}_{\mathscr{H}(k)}(\phi(z), w)=\sum_{j} \mathbb{B}_{\Omega_{n, 1}}\left(z, \Phi_{j}(w)\right) \cdot \overline{U_{j}(w)},
$$

where $u=\operatorname{det}\left(\phi^{\prime}\right)$, the $\Phi_{j}$ 's are local branches of $\phi^{-1}$, and $U_{j}=\operatorname{det}\left(\Phi_{j}^{\prime}\right)$. The Jacobian determinant of $\phi$ is given by

$$
u(z)=\operatorname{det} \phi^{\prime}(z)=\operatorname{det} \operatorname{diag}\left(\ell_{1} z_{1}^{\ell_{1}-1}, \cdots, \ell_{n} z_{n}^{\ell_{n}-1}\right)=\prod_{a=1}^{n} \ell_{a} z_{a}^{\ell_{a}-1} .
$$


The map $\phi$ has $L=\prod_{a=1}^{n} \ell_{a}$ local inverses. To enumerate them, introduce the set of multi-indices

$$
\mathfrak{B}=\left\{\left(j_{1}, \ldots, j_{n}\right) \in \mathbb{Z}^{n} \mid 0 \leq j_{a} \leq \ell_{a}-1, \text { for } a=1, \ldots, n\right\},
$$

then for each multi-index $j \in \mathfrak{B}$, there is a branch $\Phi_{j}$ of the local inverse of $\phi$ given by

$$
\Phi_{j}\left(z_{1}, \cdots, z_{n}\right)=\left(\zeta_{1}^{j_{1}} z_{1}^{\frac{1}{\ell_{1}}}, \zeta_{2}^{j_{2}} z_{2}^{\frac{1}{\ell_{2}}}, \cdots, \zeta_{n}^{j_{n}} z_{n}^{\frac{1}{\ell_{b}}}\right)
$$

where

$$
\zeta_{a}=e^{\frac{2 \pi i}{\ell_{a}}}, \quad \text { for each } 1 \leq a \leq n
$$

is an $\ell_{a}$-th root of unity, and the root functions $z_{1}^{\frac{1}{\ell_{1}}}, \ldots, z_{n}^{\frac{1}{\ell_{n}}}$ exist locally off the critical locus. We then have for each $j \in \mathfrak{B}$

$$
U_{j}(w)=\operatorname{det} \Phi_{j}^{\prime}(w)=\operatorname{det} \operatorname{diag}\left(\frac{\zeta_{1}^{j_{1}}}{\ell_{1}} w_{1}^{\frac{1}{\ell_{1}}-1}, \cdots, \frac{\zeta_{n}^{j_{n}}}{\ell_{n}} w_{n}^{\frac{1}{\ell_{n}}-1}\right)=\prod_{a=1}^{n} \frac{\zeta_{a}^{j_{a}}}{\ell_{a}} w_{a}^{\frac{1}{\ell_{a}}-1},
$$

where $\operatorname{diag}(\cdot)$ denotes a diagonal matrix with the specified diagonal entries. Therefore by Bell's formula (4.2) we have

$$
\begin{aligned}
& \prod_{a=1}^{n} \ell_{a} z_{a}^{\ell_{a}-1} \cdot \mathbb{B}_{\mathscr{H}(k)}(\phi(z), w)=\sum_{j \in \mathfrak{B}} \mathbb{B}_{\Omega_{n, 1}}\left(z, \Phi_{j}(w)\right) \cdot \overline{\prod_{a=1}^{n} \frac{\zeta_{a}^{j_{a}}}{\ell_{a}} w_{a}^{\frac{1}{\ell_{a}}-1}} \\
& =\frac{1}{\pi^{n}} \cdot \sum_{j \in \mathfrak{B}} \frac{\prod_{b=2}^{n} \bar{\zeta}_{b}^{j_{b}} z_{b} \bar{w}_{b}^{\frac{1}{\ell_{b}}}}{\left(\prod_{b=2}^{n} \bar{\zeta}_{b}^{j_{b}} z_{b}{\overline{w_{b}}}^{\frac{1}{\ell_{b}}}-\zeta_{1}^{j_{1}} z_{1}{\overline{w_{1}}}^{\frac{1}{\ell_{1}}}\right)^{2} \cdot \prod_{b=2}^{n}\left(1-\bar{\zeta}_{b}^{j_{b}} z_{b} w_{b}^{\frac{1}{\ell_{b}}}\right)^{2}} \cdot \prod_{a=1}^{n} \frac{\bar{\zeta}_{a}^{j_{a}}}{\ell_{a}}{\overline{w_{a}}}^{\frac{1}{\ell_{a}}-1},
\end{aligned}
$$

where we have used the formula in Proposition 4.1 for the Bergman kernel of $\Omega_{n, 1}$. Introduce the abbreviations

$$
r_{a}=z_{a} \bar{w}_{a}^{\frac{1}{\ell_{a}}}, \quad a=1, \ldots, n,
$$

so that we have from the above (recall that $L=\prod_{j=1}^{n} \ell_{j}$ )

$$
\begin{aligned}
\mathbb{B}_{\mathscr{H}(k)}(\phi(z), w) & =\frac{1}{\pi^{n} L^{2}} \sum_{j \in \mathfrak{B}} \frac{\prod_{a=1}^{n} \bar{\zeta}_{a}^{j_{a}} r_{a}^{1-\ell_{a}} \cdot \prod_{b=2}^{n} \bar{\zeta}_{b}^{j_{b}} r_{b}}{\left(\prod_{b=2}^{n} \bar{\zeta}_{b}^{j_{b}} r_{b}-\bar{\zeta}_{1}^{j_{1}} r_{1}\right)^{2} \prod_{b=2}^{n}\left(1-\bar{\zeta}_{b}^{j_{b}} r_{b}\right)^{2}} \\
& =\frac{1}{\pi^{n} L^{2}} \sum_{j \in \mathfrak{B}} \frac{\bar{\zeta}_{1}^{j_{1}} r_{1}^{1-\ell_{1}} \cdot \prod_{b=2}^{n} \bar{\zeta}_{b}^{2 j_{b}} r_{b}^{2-\ell_{b}}}{\left(\prod_{b=2}^{n} \bar{\zeta}_{b}^{j_{b}} r_{b}-{\overline{\zeta_{1}}}^{j_{1}} r_{1}\right)^{2} \prod_{b=2}^{n}\left(1-\bar{\zeta}_{b}^{j_{b}} r_{b}\right)^{2}} .
\end{aligned}
$$


Let $\widehat{B}\left(r_{1}, \ldots, r_{n}\right)$ denote the quantity in (4.5). We claim that the function $\widehat{B}$ of $n$ variables has the following invariance property, which will be needed later: for each $c$ with $1 \leq c \leq n$, we have

$$
\widehat{B}\left(r_{1}, \cdots, \overline{\zeta_{c}} r_{c}, \cdots, r_{n}\right)=\widehat{B}\left(r_{1}, \cdots, r_{n}\right) .
$$

To see this, notice that we have, for each $c$ with $2 \leq c \leq n$, that

$$
\begin{gathered}
\widehat{B}\left(r_{1}, r_{2}, \cdots, \overline{\zeta_{c}} r_{c}, \cdots, r_{n}\right)= \\
\frac{1}{\bar{\zeta}_{1}^{n} L^{2}} \sum_{j \in \mathfrak{B}} \frac{r_{1}^{1-\ell_{1}} \cdot \bar{\zeta}_{c}^{2\left(j_{c}+1\right)} r_{c}^{2-\ell_{c}} \cdot \prod_{\substack{2 \leq b \leq n \\
b \neq c}}{\overline{\zeta_{b}}}^{2 j_{b}} r_{b}^{2-\ell_{b}}}{\left(\bar{\zeta}_{c}^{j_{c}+1} r_{c} \prod_{\substack{2 \leq b \leq n \\
b \neq c}} \bar{\zeta}_{b}^{j_{b}} r_{b}-\bar{\zeta}_{1}^{j_{1}} r_{1}\right)^{2}\left(1-\bar{\zeta}_{c}^{j_{c}+1} r_{c}\right)^{2} \prod_{\substack{2 \leq b \leq n \\
b \neq c}}\left(1-\bar{\zeta}_{b}^{j_{b}} r_{b}\right)^{2}} .
\end{gathered}
$$

Notice that the above sum is precisely the same as $\widehat{B}\left(r_{1}, \ldots, r_{n}\right)$, since changing $j_{c}$ to $j_{c}+1$ simply amounts to a re-indexing of the sum, thanks to the fact that the $\ell_{c}$-th roots of unity form a cyclic group generated by $\zeta_{c}$.

In a similar way, $\widehat{B}\left(\overline{\zeta_{1}} r_{1}, r_{2}, \cdots, r_{n}\right)$ is precisely the same as $\widehat{B}\left(r_{1}, \ldots, r_{n}\right)$, since changing $j_{1}$ to $j_{1}+1$ simply amounts to a re-indexing of the sum, thanks to the fact that the $\ell_{1}$-th roots of unity form a cyclic group generated by $\zeta_{1}$. These two observations combined establish (4.6).

Now let

$$
\Delta=\left(\left(\prod_{b=2}^{n} r_{b}\right)^{K}-r_{1}^{K}\right)^{2} \cdot \prod_{b=2}^{n}\left(1-r_{b}^{\ell_{b}}\right)^{2}
$$

where $K=\operatorname{lcm}\left(k_{1}, \ldots, k_{n}\right)$ as in the statement of the theorem. Then we can write

$$
\begin{aligned}
& \widehat{B}\left(r_{1}, \ldots, r_{n}\right) \\
& =\frac{1}{\pi^{n} L^{2} \Delta} \sum_{j \in \mathfrak{B}}\left(\bar{\zeta}_{1}^{j_{1}} r_{1}^{1-\ell_{1}} \prod_{b=2}^{n}{\overline{\zeta_{b}}}^{2 j_{b}} r_{b}^{2-\ell_{b}} \cdot \frac{\left(\left(\prod_{b=2}^{n} r_{b}\right)^{K}-r_{1}^{K}\right)^{2}}{\left(\prod_{b=2}^{n} \bar{\zeta}_{b}^{j_{b}} r_{b}-\bar{\zeta}^{j_{1}} r_{1}\right)^{2}} \frac{\left(1-r_{b}^{\ell_{b}}\right)^{2}}{\left(1-\bar{\zeta}_{b}^{j} r_{b}\right)^{2}}\right) \\
& =\frac{1}{\pi^{n} L^{2} \Delta} \sum_{j \in \mathfrak{B}}\left(\bar{\zeta}_{1}^{j_{1}} r_{1}^{1-\ell_{1}} \prod_{b=2}^{n} \bar{\zeta}^{2 j_{b}} r_{b}^{2-\ell_{b}} \cdot\left(\sum_{\nu=0}^{K-1}\left(\prod_{b=2}^{n}{\overline{\zeta_{b}}}^{j_{b}} r_{b}\right)^{\nu}\left(\bar{\zeta}^{j_{1}} r_{1}\right)^{K-\nu-1}\right)^{2} \times\right. \\
& \left.\times \prod_{b=2}^{n}\left(\sum_{m_{b}=0}^{\ell_{b}-1}\left({\overline{\zeta_{b}}}^{j_{b}} r_{b}\right)^{m_{b}}\right)^{2}\right)
\end{aligned}
$$




$$
\begin{aligned}
& =\frac{1}{\pi^{n} L^{2} \Delta} \sum_{\alpha_{1}=0}^{2 K-2} \sum_{\alpha_{2}=0}^{2 K+2 \ell_{2}-4} \cdots \sum_{\alpha_{n}=0}^{2 K+2 \ell_{n}-4} A(\alpha) r_{1}^{\alpha_{1}+1-\ell_{1}} \prod_{b=2}^{n} r_{b}^{\alpha_{b}+2-\ell_{b}} \\
& =\frac{1}{\pi^{n} L^{2} \Delta} \sum_{\alpha_{1}=1-\ell_{1}}^{2 K-\ell_{1}-1} \sum_{\alpha_{2}=2-\ell_{2}}^{2 K+\ell_{2}-2} \cdots \sum_{\alpha_{n}=2-\ell_{n}}^{2 K+\ell_{n}-2} \widetilde{A}(\alpha) r^{\alpha},
\end{aligned}
$$

where in (4.9), for simplicity of notation, we have expressed the quantity under the summation sign in (4.8) as a (Laurent) polynomial in the $n$ variables $\left(r_{1}, \ldots, r_{n}\right)$ with coefficients $A(\alpha) \in \mathbb{C}$. In (4.10), we have re-indexed the sum, and we denote $r^{\alpha}=r_{1}^{\alpha_{1}} \ldots r_{n}^{\alpha_{n}}$. Also, $\widetilde{A}(\alpha)=A\left(\alpha_{1}+\ell_{1}-\right.$ $\left.1, \alpha_{2}+\ell_{2}-2, \ldots, \alpha_{n}+\ell_{n}-2\right)$.

Notice that (4.10) is a multi-variable polynomial in $\left(r_{1}, \ldots, r_{n}\right)$. Then, by the invariance of $\widehat{B}$ shown in (4.6), we can replace the variable $r_{a}$, with $1 \leq a \leq n$ by $\overline{\zeta_{a}} r_{a}$, and the value of the polynomial remains unchanged

$$
\begin{aligned}
& \frac{1}{\pi^{n} L^{2} \Delta} \sum_{\alpha_{1}=1-\ell_{1}}^{2 K-\ell_{1}-1} \sum_{\alpha_{2}=2-\ell_{2}}^{2 K+\ell_{2}-2} \cdots \sum_{\alpha_{n}=2-\ell_{n}}^{2 K+\ell_{n}-2} \widetilde{A}(\alpha) r^{\alpha} \\
= & \frac{1}{\pi^{n} L^{2} \Delta} \sum_{\alpha_{1}=1-\ell_{1}}^{2 K-\ell_{1}-1} \sum_{\alpha_{2}=2-\ell_{2}}^{2 K+\ell_{2}-2} \cdots \sum_{\alpha_{n}=2-\ell_{n}}^{2 K+\ell_{n}-2} \bar{\zeta}^{\alpha_{a}} \widetilde{A}(\alpha) r^{\alpha} .
\end{aligned}
$$

Looking at the difference of the two sides of the above equation, we see that for each $r=\left(r_{1}, \ldots, r_{n}\right)$ and each $1 \leq a \leq n$, we have

$$
\sum_{\alpha_{1}=1-\ell_{1}}^{2 K-\ell_{1}-1} \sum_{\alpha_{2}=2-\ell_{2}}^{2 K+\ell_{2}-2} \cdots \sum_{\alpha_{n}=2-\ell_{n}}^{2 K+\ell_{n}-2}\left(\bar{\zeta}^{\alpha_{a}}-1\right) \widetilde{A}(\alpha) r^{\alpha}=0 .
$$

This is a polynomial in $r$ which vanishes identically, so each of its coefficients is zero. This implies that for a fixed $\alpha$, the quantity $\widetilde{A}(\alpha)$ can be non-zero only if $\left(\bar{\zeta}^{\alpha_{a}}-1\right)=0$. Since this holds for each $1 \leq a \leq n$, the only terms in (4.10) that survive are the ones in which the monomial $r^{\alpha}=r_{1}^{\alpha_{1}} r_{2}^{\alpha_{2}} \ldots r_{n}^{\alpha_{n}}$ is of the form

$$
\alpha=\ell \cdot \beta=:\left(\ell_{1} \beta_{1}, \ell_{2} \beta_{2}, \ldots, \ell_{n} \beta_{n}\right),
$$

for some $\beta \in \mathbb{Z}^{n}$. From the bounds on the indices $\alpha_{c}$ in (4.10), this implies that the indices corresponding to possibly nonzero terms are the following multiples of $\ell_{c}$ :

$$
\alpha_{c}=0, \ell_{c}, \ldots, 2 K \quad \text { for each } \quad 2 \leq c \leq n \text { if } \ell_{c} \neq 1,
$$

and

$$
\alpha_{c}=1, \ldots, 2 K-1 \text { for each } 2 \leq c \leq n \text { if } \ell_{c}=1
$$

since for these (and only these) $\alpha_{c}$, we have $2-\ell_{c} \leq \alpha_{c} \leq 2 K+\ell_{c}-2$, and $\alpha_{c}$ is divisible by $\ell_{c}$. Recall here that by Lemma 4.2, the integer $K=\operatorname{lcm}\left(k_{1}, \ldots, k_{n}\right)$ is divisible by $\ell_{c}$, since we also have $K=\operatorname{lcm}\left(\ell_{1}, \ldots, \ell_{n}\right)$. Similar arguments also show that the indices $\alpha_{1}$ for which we can have possibly nonzero terms in (4.10) are

$$
\alpha_{1}=0, \ell_{1}, \ldots, 2 K-2 \ell_{1}
$$


Using the representation $\alpha=\ell \cdot \beta=\left(\ell_{1} \beta_{1}, \ldots, \ell_{n} \beta_{n}\right)$, we see that these same indices are also described by the collection $\mathfrak{G}^{*}(k)$ of $\beta \in \mathbb{Z}^{n}$ such that

$$
0 \leq \beta_{1} \leq \frac{2 K}{\ell_{1}}-2=2 k_{1}-2
$$

and for each $2 \leq b \leq n$

$$
\begin{cases}0 \leq \beta_{b} \leq \frac{2 K}{\ell_{b}}=2 k_{b} & \text { if } \ell_{b} \neq 1, \\ 1 \leq \beta_{b} \leq 2 K-1=2 k_{b}-1 & \text { if } \ell_{b}=1 .\end{cases}
$$

Notice that the set $\mathfrak{G}$ of $(1.5)$ is contained in $\mathfrak{G}^{*}(k)$. We can now write

$$
\widehat{B}\left(r_{1}, \ldots, r_{n}\right)=(\underline{4.10})=\frac{1}{\pi^{n} \cdot L^{2} \cdot \Delta} \sum_{\beta \in \mathfrak{G}^{*}(k)} \widetilde{A}(\ell \cdot \beta) r^{\ell \cdot \beta},
$$

which follows from combining equations (4.11) through (4.13).

We now proceed to compute the coefficients $\widetilde{A}(\ell \cdot \beta)$. Introduce, a set of indices $\mathfrak{C} \subset \mathbb{Z}^{n-1}$ by setting

$$
\mathfrak{C}=\left\{\left(m_{2}, \ldots, m_{n}\right) \in \mathbb{Z}^{n-1} \mid 0 \leq m_{b} \leq \ell_{b}-1 \quad \text { for } 2 \leq b \leq n\right\} .
$$

Now, in (4.8), we rewrite the first square factor as a product of two sums over indices $\nu$ and $N$ :

$$
\begin{aligned}
& \left(\sum_{\nu=0}^{K-1}\left(\prod_{b=2}^{n}{\overline{\zeta_{b}}}^{j_{b}} r_{b}\right)^{\nu}\left({\overline{\zeta_{1}}}^{j_{1}} r_{1}\right)^{K-\nu-1}\right)^{2}= \\
& \left(\sum_{\nu=0}^{K-1}\left(\prod_{b=2}^{n} \bar{\zeta}^{j_{b}} r_{b}\right)^{\nu}\left({\overline{\zeta_{1}}}^{j_{1}} r_{1}\right)^{K-\nu-1}\right)\left(\sum_{N=0}^{K-1}\left(\prod_{b=2}^{n}{\overline{\zeta_{b}}}^{j} r_{b}\right)^{N}\left(\bar{\zeta}^{j} r_{1}\right)^{K-N-1}\right) .
\end{aligned}
$$

Similarly, writing each of the other $(n-2)$ square factors $\left(\sum_{m_{b}=0}^{\ell_{b}-1}\left({\overline{\zeta_{b}}}^{j} r_{b}\right)^{m_{b}}\right)^{2}$ for $2 \leq b \leq n$ in (4.8) as a product of sums over different indices $m_{b}$ and $M_{b}$ and then expanding the products we can rewrite (4.8) as

$$
\begin{aligned}
& \widehat{B}\left(r_{1}, \ldots, r_{n}\right) \\
= & \frac{1}{\pi^{n} L^{2} \Delta} \sum_{j \in \mathfrak{B}} \sum_{m, M \in \mathfrak{C}} \sum_{\nu, N=0}^{K-1} \bar{\zeta}_{1}^{j_{1}(2 K-\nu-N-1)} r_{1}^{2 K-\nu-N-\ell_{1}-1} \prod_{b=2}^{n} \bar{\zeta}_{b}^{j_{b}\left(\nu+N+m_{b}+M_{b}+2\right)} r_{b}^{m_{b}+M_{b}+\nu+N-\ell_{b}+2},
\end{aligned}
$$

where, in the sum above, $j=\left(j_{1}, \ldots, j_{n}\right)$ ranges over the set $\mathfrak{B}$ of (4.3), and $m=\left(m_{2}, \ldots, m_{n}\right)$ and $M=\left(M_{2}, \ldots, M_{n}\right)$ are multi-indices that range over the set $\mathfrak{C}$ of (4.17), and the indices $\nu$ and $N$ each go independently from 0 to $K-1$. To find $\widetilde{A}(\ell \cdot \beta)$, note that in the sum (4.18), we are considering those terms in which the power of $r_{1}$ is $\ell_{1} \beta_{1}$ and the power of $r_{b}$ is $\ell_{b} \beta_{b}$ for $2 \leq b \leq n$. Notice that for these powers of $r_{j}$, the powers of $\zeta_{j}$ 's are each 1 . Therefore, comparing the two expressions (4.18) and (4.16) for $\widehat{B}\left(r_{1}, \ldots, r_{n}\right)$, we conclude that for each $\beta \in \mathfrak{G}^{*}(k)$ we have

$$
\widetilde{A}(\ell \cdot \beta)=\sum^{\prime} \bar{\zeta}^{j_{1}(2 K-\nu-N-1)} \prod_{b=2}^{n} \bar{\zeta}_{b}^{j_{b}\left(\nu+N+m_{b}+M_{b}+2\right)}
$$




$$
=\sum^{\prime} 1
$$

where $\sum^{\prime}$ denotes a sum extending over the set of indices $j=\left(j_{1}, j_{2}, \ldots, j_{n}\right), m=\left(m_{2}, \ldots, m_{n}\right), M=$ $\left(M_{2}, \ldots, M_{n}\right)$ and $\nu, N$ ranging over

$$
\left\{\begin{array}{l}
j \in \mathfrak{B}, m, M \in \mathfrak{C} \\
0 \leq \nu, N \leq K-1 \\
m_{b}+M_{b}+\nu+N+2-\ell_{b}=\beta_{b} \ell_{b}, \quad \text { for each } \quad 2 \leq b \leq n \\
2 K-\nu-N-\ell_{1}-1=\beta_{1} \ell_{1} .
\end{array}\right.
$$

The expression in (4.20) follows from (4.19) since for each such index, the summand is clearly 1. Observe now that in the range of summation described above, the indices $j=\left(j_{1}, j_{2}, \ldots, j_{n}\right) \in \mathfrak{B}$ (with $\mathfrak{B}$ as in (4.3) ) vary freely without any interaction with the other indices $m, M, \nu, N$. Therefore,

$$
\widetilde{A}(\ell \cdot \beta)=(\underline{4.20})=\sum_{j \in \mathfrak{B}} C(\beta)=|\mathfrak{B}| \cdot C(\beta)=L \cdot C(\beta),
$$

where as in the statement of the theorem, $L=\prod_{a=1}^{n} \ell_{a}$, and $C(\beta)$ is the number of solutions in integers $m=\left(m_{2}, \ldots, m_{n}\right), M=\left(M_{2}, \ldots, M_{n}\right), \nu, N$ of the system of equations and inequalities given by

$$
\begin{cases}0 \leq m_{b}, M_{b} \leq \ell_{b}-1, & \text { for each } 2 \leq b \leq n \\ 0 \leq \nu, N \leq K-1, & \\ m_{b}+M_{b}+\nu+N=\ell_{b}\left(\beta_{b}+1\right)-2 & \text { for each } 2 \leq b \leq n \\ \nu+N=2 K-\ell_{1}\left(\beta_{1}+1\right)-1 . & \end{cases}
$$

To find $C(\beta)$, we first note that the third equation may be replaced (with the help of the last equation) by the equivalent equation

$$
m_{b}+M_{b}=\ell_{b}\left(\beta_{b}+1\right)+\ell_{1}\left(\beta_{1}+1\right)-2 K-1 \text { for each } 2 \leq b \leq n .
$$

Consequently, the number of solutions $C(\beta)$ of the system can be obtained by multiplying together the number of solutions of

$$
\nu+N=2 K-\ell_{1}\left(\beta_{1}+1\right)-1, \quad 0 \leq \nu, N \leq K-1
$$

with the number of solutions for each $b$, with $2 \leq b \leq n$ to

$$
m_{b}+M_{b}=\ell_{b}\left(\beta_{b}+1\right)+\ell_{1}\left(\beta_{1}+1\right)-2 K-1, \quad 0 \leq m_{b}, M_{b} \leq \ell_{b}-1 .
$$

To represent these numbers, for integers $\lambda$, $\mu$, define $D_{\lambda}(\mu)$ to be the number of integer solutions $(x, y) \in \mathbb{Z}^{2}$ of the system of equations and inequalities:

$$
\begin{aligned}
& x+y=\mu, \\
& 0 \leq x \leq \lambda-1, \\
& 0 \leq y \leq \lambda-1 .
\end{aligned}
$$

Then clearly we have

$$
C(\beta)=\mathrm{D}_{K}\left(2 K-\ell_{1}\left(\beta_{1}+1\right)-1\right) \cdot \prod_{b=2}^{n} \mathrm{D}_{\ell_{b}}\left(\ell_{b}\left(\beta_{b}+1\right)+\ell_{1}\left(\beta_{1}+1\right)-2 K-1\right) .
$$


Claim: the numbers $\mathrm{D}_{\lambda}(\mu)$ are given by the formula (1.2) that precedes the statement of Theorem 1.1

Indeed, if $\mu \leq-1$, then by (4.23), we have $x+y \leq-1$. However, from (4.24) and (4.25) in the definition of $\mathrm{D}_{\lambda}(\mu)$, this is impossible. Hence, $\mathrm{D}_{\lambda}(\mu)=0$. Similarly, if $\mu \geq 2 \lambda-1$, then by (4.23), $x+y \geq 2 \lambda-1$. However, from (4.24) and (4.25) in the definition of $\mathrm{D}_{\lambda}(\mu)$, this is impossible. Hence, $\mathrm{D}_{\lambda}(\mu)=0$.

In the other cases, it is easy to enumerate the solutions. If $0 \leq \mu \leq \lambda-1$, then

$$
\mathrm{D}_{\lambda}(\mu)=|\{(x, \mu-x): 0 \leq x \leq \mu\}|=\mu+1,
$$

and if $\lambda \leq \mu \leq 2 \lambda-2$, then

$$
\mathrm{D}_{\lambda}(\mu)=|\{(x, \mu-x): \mu-\lambda+1 \leq x \leq \lambda-1\}|=2 \lambda-1-\mu,
$$

completing the proof of the claim.

From (4.16) and (4.21) we see that

$$
\mathbb{B}_{\mathscr{H}(k)}(\phi(z), w)=\widehat{B}\left(r_{1}, \ldots, r_{n}\right)=\frac{1}{\pi^{n} L^{2} \Delta} \sum_{\beta \in \mathfrak{G}^{*}(k)} L \cdot C(\beta) r^{\ell \cdot \beta}=\frac{1}{\pi^{n} L \Delta} \sum_{\beta \in \mathfrak{G}^{*}(k)} C(\beta) r^{\ell \cdot \beta} .
$$

Now

$$
\phi(z)=\left(\phi_{1}(z), \ldots, \phi_{n}(z)\right)=\left(z_{1}^{\ell_{1}}, \ldots, z_{n}^{\ell_{n}}\right) .
$$

Therefore, recalling the definition (4.4), we see that

$$
\begin{aligned}
r^{\ell \cdot \beta} & =\left(r_{1}^{\ell_{1}}\right)^{\beta_{1}} \cdots\left(r_{n}^{\ell_{n}}\right)^{\beta_{n}} \\
& =\left(z_{1}^{\ell_{1}} \overline{w_{1}}\right)^{\beta_{1}} \ldots\left(z_{n}^{\ell_{n}} \overline{w_{n}}\right)^{\beta_{n}} \\
& =\left(\phi_{1}(z) \overline{w_{1}}\right)^{\beta_{1}} \cdots\left(\phi_{n}(z) \overline{w_{n}}\right)^{\beta_{n}} .
\end{aligned}
$$

Also, remembering that $\ell_{b}=\frac{K}{k_{b}}$ for each $b$, we have

$$
r_{b}^{K}=\left(z_{b}^{\ell_{b}}\right)^{k_{b}}{\overline{w_{b}}}^{k_{b}}=\phi_{b}(z)^{k_{b}}{\overline{w_{b}}}^{k_{b}},
$$

and

$$
r_{b}^{\ell_{b}}=z_{b}^{\ell_{b}} \overline{w_{b}}=\phi_{b}(z) \overline{w_{b}} .
$$

Therefore, recalling the definition (4.7), we have

$$
\begin{aligned}
\Delta & =\left(\left(\prod_{b=2}^{n} r_{b}\right)^{K}-r_{1}^{K}\right)^{2} \cdot \prod_{b=2}^{n}\left(1-r_{b}^{\ell_{b}}\right)^{2} \\
& =\left(\left(\prod_{b=2}^{n} \phi_{b}(z)^{k_{b}} \bar{w}_{b} k_{b}\right)-\phi_{1}(z)^{k_{1}}{\overline{w_{1}}}^{k_{1}}\right)^{2} \cdot \prod_{b=2}^{n}\left(1-\phi_{b}(z) \overline{w_{b}}\right)^{2} .
\end{aligned}
$$

Therefore, if we replace $\phi(z)$ by $z$ in the first member of (4.27), we see that the last member is transformed to a function of $\left(t_{1}, \ldots, t_{n}\right)$, where $t_{a}=z_{a} \overline{w_{a}}$. In fact, we get (1.3), thus completing the proof of the result, except that in the numerator of (1.3) we have obtained the polynomial $\sum_{\beta \in \mathfrak{G}^{*}(k)} C(\beta) t^{\beta}$ instead of $\sum_{\beta \in \mathfrak{G}} C(\beta) t^{\beta}$. Therefore, to complete the proof, we need to show that if $\beta \in \mathfrak{G}^{*}(k) \backslash \mathfrak{G}$ then $C(\beta)=0$. Now for such a $\beta$, there exists a $2 \leq b \leq n$ such that $\ell_{b}=1$ and $\beta_{b}$ is either 0 or $2 k_{b}$. First assume that $\beta_{b}=0$. Then the factor $\mathrm{D}_{\ell_{b}}\left(\ell_{b}\left(\beta_{b}+1\right)+\ell_{1}\left(\beta_{1}+1\right)-2 K-1\right)$ 
in the formula (1.4) reduces to $D_{1}\left(\ell_{1}\left(\beta_{1}+1\right)-2 K\right)$. By the definition (1.2) of $D$, this is not zero if and only if $\ell_{1}\left(\beta_{1}+1\right)-2 K=0$. However, in the latter case, we have the first factor of (1.4) equal to zero, since it equals $\mathrm{D}_{K}(-1)$.

In the other case $\beta_{b}=2 k_{b}=2 K$ we see that the factor $\mathrm{D}_{\ell_{b}}\left(\ell_{b}\left(\beta_{b}+1\right)+\ell_{1}\left(\beta_{1}+1\right)-2 K-1\right)$ reduces to $D_{1}\left(\ell_{1}\left(\beta_{1}+1\right)\right)=0$.

4.3. Recapturing the special cases $\mathscr{H}(1,-k)$ and $\mathscr{H}(k,-1)$. We now show that the results of [Edh16] on explicit Bergman kernels of fat and thin Hartogs triangles are special cases of Theorem 1.1 .

4.3.1. $\mathscr{H}(1,-k), k \geq 1$. We follow the notation used in Theorem 1.1. For $\mathscr{H}(1,-k)$ we have $k_{1}=1$ and $k_{2}=k$. Hence $K=\operatorname{lcm}(1, k)=k$ and $L=k$. We then have

$$
\mathfrak{G}=\left\{\left(\beta_{1}, \beta_{2}\right) \in \mathbb{Z}^{2} \mid \beta_{1}=0,0 \leq \beta_{2} \leq 2 k\right\} .
$$

For $\left(0, \beta_{2}\right) \in \mathfrak{G}$, we compute $C\left(0, \beta_{2}\right)$, where $0 \leq \beta_{2} \leq 2 k$. By (1.4), we have

$$
C\left(0, \beta_{2}\right)=\mathrm{D}_{k}(k-1) \mathrm{D}_{1}\left(\beta_{2}-k\right) .
$$

Now from (1.2), we have $\mathrm{D}_{k}(k-1)=k$ and

$$
\mathrm{D}_{1}\left(\beta_{2}-k\right)= \begin{cases}0 & 0 \leq \beta_{2} \leq k-1 \\ 1 & \beta_{2}=k \\ 0 & k+1 \leq \beta_{2} \leq 2 k\end{cases}
$$

Hence for $\beta=\left(0, \beta_{2}\right) \in \mathfrak{G}, C(\beta) \neq 0$ if and only if $\beta_{2}=k$ and in this case, $C(\beta)=k$. Hence the formula (1.3) gives

$$
\mathbb{B}_{\mathscr{H}(1,-k)}(z, w)=\frac{1}{\pi^{2} k} \cdot \frac{k t_{2}^{k}}{\left(t_{2}^{k}-t_{1}\right)^{2}\left(1-t_{2}\right)^{2}}=\frac{1}{\pi^{2}} \cdot \frac{t_{2}^{k}}{\left(t_{2}^{k}-t_{1}\right)^{2}\left(1-t_{2}\right)^{2}},
$$

which precisely is the content of [Edh16, Theorem 1.4].

4.3.2. $\mathscr{H}(k,-1), k \geq 2$. In this case, $k_{1}=k$ and $k_{2}=1$. Hence $K=k$ and $L=k$. We then have

$$
\mathfrak{G}=\left\{\left(\beta_{1}, \beta_{2}\right) \in \mathbb{Z}^{2} \mid 0 \leq \beta_{1} \leq 2 k-2,0 \leq \beta_{2} \leq 2\right\},
$$

and

$$
C(\beta)= \begin{cases}\mathrm{D}_{k}\left(2 k-\beta_{1}-2\right) \mathrm{D}_{k}\left(\beta_{1}-k\right), & \beta=\left(\beta_{1}, 0\right) \\ \mathrm{D}_{k}\left(2 k-\beta_{1}-2\right) \mathrm{D}_{k}\left(\beta_{1}\right), & \beta=\left(\beta_{1}, 1\right) \\ \mathrm{D}_{k}\left(2 k-\beta_{1}-2\right) \mathrm{D}_{k}\left(\beta_{1}+k\right), & \beta=\left(\beta_{1}, 2\right)\end{cases}
$$

We compute $\mathrm{D}_{k}$ 's.

$$
\begin{gathered}
\mathrm{D}_{k}\left(2 k-\beta_{1}-2\right)= \begin{cases}\beta_{1}+1, & 0 \leq \beta_{1} \leq k-1 \\
2 k-\beta_{1}-1, & k \leq \beta_{1} \leq 2 k-2 .\end{cases} \\
\mathrm{D}_{k}\left(\beta_{1}-k\right)= \begin{cases}0, & 0 \leq \beta_{1} \leq k-1 \\
\beta_{1}-k+1, & k \leq \beta_{1} \leq 2 k-2\end{cases}
\end{gathered}
$$




$$
\begin{aligned}
\mathrm{D}_{k}\left(\beta_{1}\right) & = \begin{cases}\beta_{1}+1, & 0 \leq \beta_{1} \leq k-1 \\
2 k-1-\beta_{1}, & k \leq \beta_{1} \leq 2 k-2 .\end{cases} \\
\mathrm{D}_{k}\left(\beta_{1}+k\right) & = \begin{cases}k-\beta_{1}-1, & 0 \leq \beta_{1} \leq k-1 \\
0, & k \leq \beta_{1} \leq 2 k-2 .\end{cases}
\end{aligned}
$$

Hence,

$$
\begin{aligned}
& \sum_{\beta \in \mathfrak{G}} C(\beta) t^{\beta}=\underbrace{\sum_{\beta_{1}=k}^{2 k-2}\left(2 k-\beta_{1}-1\right)\left(\beta_{1}-k+1\right) t_{1}^{\beta_{1}}}_{\beta_{2}=0}+\underbrace{\left(\sum_{\beta_{1}=0}^{k-1}\left(\beta_{1}+1\right)^{2} t_{1}^{\beta_{1}} t_{2}+\sum_{\beta_{1}=k}^{2 k-2}\left(2 k-\beta_{1}-1\right)^{2} t_{1}^{\beta_{1}} t_{2}\right)}_{\beta_{2}=1} \\
& +\underbrace{\sum_{\beta_{1}=0}^{k-1}\left(\beta_{1}+1\right)\left(k-\beta_{1}-1\right) t_{1}^{\beta_{1}} t_{2}^{2}}_{\beta_{2}=2}
\end{aligned}
$$

We rewrite the terms corresponding to $\beta_{2}=0,1,2$ as follows. In the term for $\beta_{2}=0$, by making the substitution $\ell=\beta_{1}-k+1$, we obtain $\left(\sum_{\ell=1}^{k-\ell}(k-\ell) \ell \cdot t_{1}^{\ell-1}\right) t_{1}^{k}$.

In the first sum of the second term (which corresponds to $\beta_{2}=1$ ) we make the substitution $\ell=\beta_{1}+1$, which transforms it into $\sum_{\ell=1}^{k} \ell^{2} \cdot t_{1}^{\ell-1} t_{2}$. In the second sum, we make the substitution $\ell=\beta_{1}-k+1$, which transforms it into $\sum_{\ell=1}^{k}(k-\ell)^{2} \cdot t_{1}^{k+\ell-1} t_{2}$. Combining the two we can represent the second term as

$$
\left.\sum_{\ell=1}^{k} \ell^{2} t_{1}^{\ell-1} t_{2}+\sum_{\ell=1}^{k}(k-\ell)^{2} t_{1}^{k+\ell-1} t_{2}=\left(\sum_{\ell=1}^{k}\left(\ell^{2}+(k-\ell)^{2}\right) t_{1}^{k}\right) t_{1}^{\ell-1}\right) t_{2} .
$$

Similarly using the substitution $\ell=\beta_{1}+1$, the last term becomes

$$
\sum_{\beta_{\ell}=0}^{k-1}\left(\beta_{1}+1\right)\left(k-\beta_{1}-1\right) t_{1}^{\beta_{1}} t_{2}^{2}=\sum_{\ell=1}^{k} \ell(k-\ell) t_{1}^{\ell-1} t_{2}^{2} .
$$

Therefore we get the expression for the Bergman kernel for $\mathscr{H}(k,-1)$ as

$$
\frac{1}{\pi^{2} k} \cdot \frac{\left.\left(\sum_{\ell=1}^{k-1}(k-\ell) \cdot \ell \cdot t_{1}^{\ell-1}\right) t_{1}^{k}+\left(\sum_{\ell=1}^{k}\left(\ell^{2}+(k-\ell)^{2}\right) t_{1}^{k}\right) t_{1}^{\ell-1}\right) t_{2}+\left(\sum_{\ell=1}^{k} \ell(k-\ell) t_{1}^{\ell-1}\right) t_{2}^{2}}{\left(t_{2}-t_{1}^{k}\right)^{2}\left(1-t_{2}\right)^{2}} .
$$

The above expression is precisely the statement of [Edh16, Theorem 1.2]. 


\section{REFERENCES}

[Beh33] Heinrich Behnke. Zur Theorie der Singularitäten der Funktionen mehrerer komplexen Veränderlichen. Mathematische Annalen, 108(1):91-104, 1933.

[Bel82] Steven R. Bell. The Bergman kernel function and proper holomorphic mappings. Trans. Amer. Math. Soc., 270(2):685-691, 1982.

[Bel05] Steven R. Bell. The Bergman kernel and quadrature domains in the plane. In Quadrature domains and their applications, volume 156 of Oper. Theory Adv. Appl., pages 61-78. Birkhäuser, Basel, 2005.

[Bre55] H. J. Bremermann. Holomorphic continuation of the kernel function and the Bergman metric in several complex variables. In Lectures on functions of a complex variable, pages 349-383. The University of Michigan Press, Ann Arbor, 1955.

[Cat80] David Catlin. Boundary behavior of holomorphic functions on pseudoconvex domains. J. Differential Geom., 15(4):605-625 (1981), 1980.

[CEM19] D. Chakrabarti, L. D. Edholm, and J. D. McNeal. Duality and approximation of Bergman spaces. Adv. Math., 341:616-656, 2019.

[Cha18] Debraj Chakrabarti. On an observation of Sibony. arXiv e-prints; To appear in Proc. of the Amer. Math.Soc., page arXiv:1807.05277, Jul 2018.

[Che17] Liwei Chen. The $L^{p}$ boundedness of the Bergman projection for a class of bounded Hartogs domains. J. Math. Anal. Appl., 448(1):598-610, 2017.

[CKY19] Liwei Chen, Steven G. Krantz, and Yuan Yuan. $L^{p}$ regularity of the Bergman Projection on domains covered by the polydisk. arXiv e-prints, page arXiv:1903.10497, Mar 2019.

[CZ16] Debraj Chakrabarti and Yunus E. Zeytuncu. $L^{p}$ mapping properties of the Bergman projection on the Hartogs triangle. Proc. Amer. Math. Soc., 144(4):1643-1653, 2016.

[DS04] Peter Duren and Alexander Schuster. Bergman spaces, volume 100 of Mathematical Surveys and Monographs. American Mathematical Society, Providence, RI, 2004.

[Edh16] Luke D. Edholm. Bergman theory of certain generalized Hartogs triangles. Pacific J. Math., 284(2):327-342, 2016.

[EM16] L. D. Edholm and J. D. McNeal. The Bergman projection on fat Hartogs triangles: $L^{p}$ boundedness. Proc. Amer. Math. Soc., 144(5):2185-2196, 2016.

[EM17] L. D. Edholm and J. D. McNeal. Bergman subspaces and subkernels: degenerate $L^{p}$ mapping and zeroes. $J$. Geom. Anal., 27(4):2658-2683, 2017.

[FS09] Philippe Flajolet and Robert Sedgewick. Analytic combinatorics. Cambridge University Press, Cambridge, 2009.

[HS80] Monique Hakim and Nessim Sibony. Spectre de $A(\bar{\Omega})$ pour des domaines bornés faiblement pseudoconvexes réguliers. J. Funct. Anal., 37(2):127-135, 1980.

[Huo18] Zhenghui Huo. $L^{p}$ estimates for the Bergman projection on some Reinhardt domains. Proc. Amer. Math. Soc., 146(6):2541-2553, 2018.

[JP08] Marek Jarnicki and Peter Pflug. First steps in several complex variables: Reinhardt domains. EMS Textbooks in Mathematics. European Mathematical Society (EMS), Zürich, 2008.

[Kra13] Steven G. Krantz. Geometric analysis of the Bergman kernel and metric, volume 268 of Graduate Texts in Mathematics. Springer, New York, 2013.

[Par18] Jong-Do Park. The explicit forms and zeros of the Bergman kernel for 3-dimensional Hartogs triangles. J. Math. Anal. Appl., 460(2):954-975, 2018.

[Ran86] R. Michael Range. Holomorphic functions and integral representations in several complex variables, volume 108 of Graduate Texts in Mathematics. Springer-Verlag, New York, 1986.

[Sha15] Mei-Chi Shaw. The Hartogs triangle in complex analysis. In Geometry and topology of submanifolds and currents, volume 646 of Contemp. Math., pages 105-115. Amer. Math. Soc., Providence, RI, 2015.

[Sib75] Nessim Sibony. Prolongement des fonctions holomorphes bornées et métrique de Carathéodory. Invent. Math., 29(3):205-230, 1975. 
E-mail address: chakr2d@cmich.edu

URL: http://people.cst.cmich.edu/chakr2d/

E-mail address: konke1am@cmich.edu

E-mail address: maink1m@cmich.edu

$U R L:$ http://people.cst.cmich.edu/maink1m/

E-mail address: mille7em@cmich.edu

Department of Mathematics, Central Michigan University, Mt. Pleasant, Mi 48859, USA 